\title{
Relationship between PPI and baseline startle response
}

\author{
Guy Sandner $\cdot$ Nathalie Monique Canal
}

Received: 26 April 2006/ Accepted: 27 August 2006/Published online: 31 October 2006

(C) Springer Science+Business Media B.V. 2006

\begin{abstract}
Prepulse inhibition (PPI) of the startle response to a sudden noise is the reduction in startle observed when the noise is preceded shortly by a mild sensory event, which is often a tone. A part of the literature is based on the assumption that PPI is independent of the baseline startle. A simple model is presented and experimental validation provided. The model is based on the commonly accepted observation that the neuronal circuit of PPI differs from that of startle. But, by using a common output, the measures of both phenomena become linked to each other. But, how can we interpret the numerous experimental data showing PPI to be independent of the startle level? It is suggested that in a number of such cases the baseline startle would have been stabilized by a ceiling effect in the startle/PPI neuronal networks. Reducing the startle level, for example in a PPI evaluation procedure, may disclose properties of startle masked by this ceiling effect. Disclosure of habituation to the startle eliciting noise produced an increase of PPI along its initial measurements. Taken together, even if the neuronal process that sustains startle and PPI are distinct, separating them experimentally requires careful parametric methods and caution in the interpretation of the corresponding observations.
\end{abstract}

Keywords Audition - Startle $\cdot$ Habituation · Prepulse inhibition $\cdot$ Model $\cdot$ Schizophrenia

G. Sandner $(\bowtie) \cdot$ N. M. Canal

I.N.S.E.R.M. U666, Faculté de médecine, Université Louis Pasteur, 11, Rue Humann, 67085 Strasbourg Cedex, France e-mail: sandner@neurochem.u-strasbg.fr

\section{Introduction}

Some behavioral features altered in schizophrenia can be tested in both human and lower animal species. One of them is "prepulse inhibition of the startle reflex" (PPI) (Braff et al. 2001). A burst of noise triggers a muscle twitch known as the acoustic startle response (Davis 1970, 1974). This response is reduced when the startle-inducing noise is preceded by a mild stimulus, called prepulse, that does not induce a startle response on its own (Groves et al. 1974; Graham 1975; Sanford et al. 1992). Deficits in PPI observed in patients and elicited experimentally in lower animals have been used to gauge the efficiency of anti-psychotic drugs (Geyer et al. 2001; Swerdlow and Geyer 1998).

PPI, is assessed by a decrease of the amplitude of the startle response. But, its independence of the baseline startle is questionable. It was believed to be guaranteed by computing the following ratio: $100 \times($ baseline startle - startle after a prepulse)/baseline startle. This assumption was reinforced by observations showing that drugs altered PPI without affecting the baseline startle level (Johansson et al. 1995). But such observations did not fit with predictions obtained by a model of PPI. Two models of startle and PPI have been published. Leumann et al. (2001) modeled the neuronal pathways of startle and PPI (Koch 1999). They simulated the properties of its components with a computer program, and estimated their impact on the startle and PPI. The complexity of the architecture of the neuronal pathways was "increased up to a level that was necessary to obtain realistic functional properties". But, the relationship between PPI and baseline startle level had not been questioned. In another model, Schmajuk and Larrauri (2005) and Schmajuk et al. (2006) simulated 
also startle and PPI. They simulated the properties of a number of functional modules whose output interacted to produce the startle response, their activity being triggered by sounds. The modules differed from each other in terms of transfer function, delay and time course. Sounds elicited an exponential tonic and phasic excitation interacting with a delayed linear inhibition. This was modeled by mathematical equations with parameters adjusted so that the simulated startle and PPI fitted with most experimental data in the literature. In a chapter entitled "predictions of the model", Schmajuk and Larrauri (2005) wrote that "an identical absolute reduction in responding produces a greater proportional decrease for weaker pulses than for stronger ones". This means that PPI would depend on the baseline startle level. We decided to verify this possibility with a slightly different and simpler theoretical approach. We simulated startle and PPI, in some sense like Schmajuk et al, but incorporating the fluctuating nature of the iterative startle measurements. This was done because we suspected some computational artifacts that would pollute the computations. Indeed, the input-output relationship is seldom a linear one in neuronal networks. After description of the model, its corresponding prediction on PPI and baseline startle levels was submitted to an experimental verification. The latter consisted of standard PPI computations obtained from individual startles provided by the model and by an animal study. This was a direct validation of the idea that PPI and baseline startle level could not be considered apart from each other. But, this seems in contradiction with numerous studies reporting PPI changes without change of the baseline startle level. For solving this contradiction, we suggest that the baseline startle may have been stabilized by a ceiling effect. Such effect could express the property of a functional module that participates in the generation of the startle reaction. Reduction in its level of activation by the prepulse would lower this from that of the ceiling level. A second series of behavioral experiments was conducted to test this idea. We considered habituation which startle is prone to (Groves and Thompson 1970). The startle eliciting stimulation parameters were set so as to keep habituation hidden. If our supposition is valid, PPI, by reducing the startle level would allow habituation to become visible.

This paper represents two approaches to the same question: (i) a simulation with its experimental validation and (ii) a new explanation of known results and their extension. We decided to present the arguments and data in a stepwise manner, each one preceded by its specific introduction and followed by a brief intermediary discussion.

\section{Computational model}

Introduction: justification of what has been simulated

The sudden activation of para-vertebral muscles characteristic of the startle response corresponds to a burst of activity in giant neurons of nucleus pontis caudalis. It is directly triggered by an activation of the cochlear root nuclei when a strong and unpredicted noise occurs (Nodal and Lopez 2003). Membrane depolarization of giant neurons is mediated by calcium channels, triggered by glutamate-NMDA receptors (Krase et al. 1993). It is otherwise modulated by several influences, among which an inhibitory one coming from the pedunculopontine tegmental nucleus. This pathway is activated by most sensory stimuli (auditory, visual, tactile) (Yeomans and Frankland 1995). These pedunculopontine neurons are cholinergic. They inhibit nucleus pontis caudalis giant neurons through muscarinic receptors (Koch et al. 1993). Muscarinic receptors down regulate giant neuron responsiveness by decreasing its adenyl cyclase activity (de Lima and Davis 1995; van Koppen and Kayser 2003). Taken together, the startle inducing noise should elicit a strong depolarization of giant neurons of the nucleus pontis caudalis, whereas the prepulses should tune down their sensitivity. It also means that the baseline startle and PPI are carried over by independent neuronal networks which converge on pedunculopontine neurons and interact there. This is what the Schmajuk and Larrauri (2005) and Schmajuk et al.'s model (2006) expresses. Other, uncontrolled influences set the membrane potential of such neurons to various levels, differing from one moment to another and from one animal to another. This has to be considered as it results in some startle reactions being strong, and others weak.

Simulation methods

\section{Principle}

Responsiveness of peduncolopontine neurons to strong sounds was modeled by a nonlinear transfer function, comprising a linear part, limited by a threshold and a ceiling. This point differed from Schmajuk and Larrauti (2005) and Schmajuk et al.'s model (2006). It expressed the fact that neuronal cell membrane fluctuations are limited respectively by potassium channel and calcium channel potentials. Uncontrolled fluctuating influences on penduncolopontine neurons were simulated by changing the offset of the startling 
acoustic signal. The inhibitory influence produced by the prepulses was modeled by an attenuation of the mean slope of the transfer function.

\section{Computations}

We assumed that any input startling stimulus activates more or less the motor response according to an arc tangent input-output transfer function. We computed transforms of the inputs. Individual startles were drawn out of a Gaussian distribution. Their transformed values were evaluated. Means of these values were computed. The transfer function change, representing the inhibitory effect of a prepulse, was obtained by attenuating the input influence in a linear way, computed as follows: input with prepulse $=$ input without prepulse/2-1 (see Fig. 1). More details on the methods are given in the legend of Fig. 1.

\section{Modeled data}

Bar graphs in the top right of Fig. 1 show the mean startle levels obtained with three levels of inputs. Startle levels were not proportional to the input levels. For strong auditory inputs, and subsequent high levels of startle, the latter varied less. Below these bar graphs, the computed percent PPI was represented. PPI levels depended on the baseline startle. When startles came close to their ceiling, PPIs decreased substantially. A strong PPI decline was correlated with a weak increase of startle.

Discussion of the modeled data

There is only one model to which such data can be compared (Schmajuk and Larrauri 2005; Schmajuk et al. 2006). The conclusion that results from our computations is similar to those of this model, except that the startle levels varied much less in our model because these authors used a linear and log transform function respectively for the baseline startle and PPI. It is relevant to focus on the medium to strong startling condition since the most recent papers provided experimental data obtained with strong startling noises $(120 \mathrm{~dB}(\mathrm{~A}))$. Furthermore the test conditions were stressful which is known to enhance the reactivity of the neuronal startle circuit (Davis 1989; Le Pen and Moreau 2002; Lipska et al. 1995). PPI was reported to vary but the baseline startle did not. For these reasons we speculated that in such PPI measurement experiments, the baseline startle could have been stabilized by some ceiling effect.
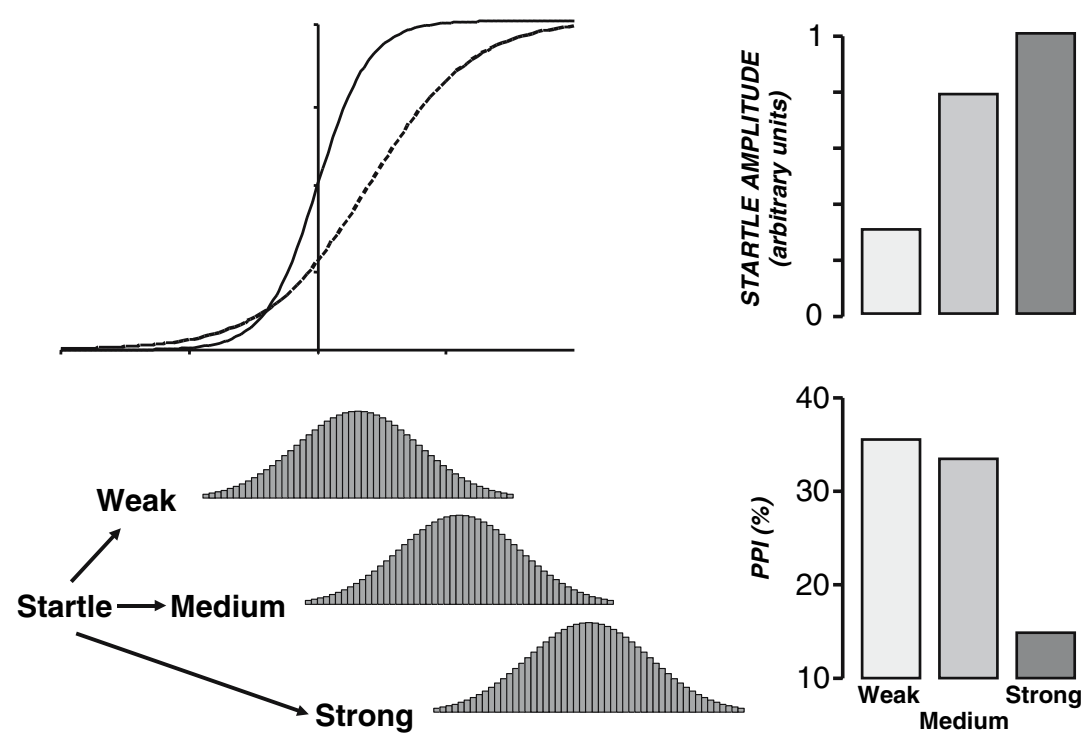

Fig. 1 Illustration of the model construct. The graph at the top of the page represents two transfer functions. The full line holds for the baseline startle transfer function. The dotted line corresponded to the same equation, but combined with the linear attenuation reported in the simulation methods section. Below the $x$-axis (input stimuli), there are three identical Gaussian input distribution, each with its specific mean value.
They modeled respectively weak, medium, and strong startle trigger inputs. The $y$-axis (outputs) values were used to compute the resulting distributions from which mean startles and percent PPI were calculated. At the right of the figure, bar graphs represent the resulting mean startle levels (top graph) and percent PPI (bottom graph), respectively, for the weak, medium, and strong inputs 


\section{Comparing the model to actual experimental data}

Introduction

This behavioral experiment consisted in computing actual experimental data in the same way as in the model. The data were obtained in an experiment using the same strong auditory stimuli as in recent papers on "animal models of schizophrenia" (Le Pen and Moreau 2002; Lipska et al. 1995). The level of stress of the rats was assumed to be the same as in this literature, rats being also placed in a narrow tube.

Methods common to all behavioral experiments

The study was performed by researchers, all of whom being authorized to manipulate living animals by the French Ministry of Agriculture. Procedures conformed to institutional guidelines, which comply with the European Communities Council Directive of November 24, 1986 (86/609/EEC) and the National Council Directive of October 19, 1987 (87848-Ministry of Agriculture and Forestry, Veterinary Health and Animal Protection Department). All efforts were made to avoid animal suffering and reduce the number of animals used.

\section{Animals}

Forty-seven male Sprague Dawley rats, weighing 250 $279 \mathrm{~g}, 2$-month-old, were used for this first experiment (Janvier, France). One hundred and forty-eight male Long-Evans rats, weighing 270-350 g, were used for the other experiments (Janvier, France). They were new to startle experiments. They were kept on a constant light/dark cycle (light from 7 a.m. to 9 p.m.) and housed two per cage, with free access to food and water. All experiments took place between 9 a.m. and 5 p.m.

\section{PPI apparatus}

Rats were confined for testing either in a contention tube (diameter $6 \mathrm{~cm}$, length $20 \mathrm{~cm}$ ) for the first experiment, or were allowed to move freely in a transparent cage $(26 \mathrm{~cm} \times 25 \mathrm{~cm} \times 18 \mathrm{~cm})$ for the other experiments. They were placed in a soundattenuating chamber. The background noise was produced by a noise generating electronic device $66 \mathrm{~dB}(\mathrm{~A})$. A PC was used to trigger two types of acoustic stimuli, a pure tone $(75 \mathrm{~dB}(\mathrm{~A})$ in the first experiment and $85 \mathrm{~dB}(\mathrm{~A})$ in the other experiments,
$3,000 \mathrm{~Hz}$, duration $20 \mathrm{~ms}$ ) or a wide band noise (120 dB(A), first experiment; $105 \mathrm{~dB}(\mathrm{~A})$, other experiments; bandwidth: $20 \mathrm{~Hz}-20 \mathrm{kHz}$, duration $50 \mathrm{~ms}$ ), independently amplified and transmitted to each of two loudspeakers. The floor of the test cage was a platform fixed to an electronic scale. Its force transducer was connected to an amplifier (AM502, Tektronix, Gain: 1000, bandwidth: $0.1 \mathrm{~Hz}-1 \mathrm{kHz}$ ), which supplied a voltage proportional to the force to an A/D converter (AD800, Analog Devices) fitted in the computer. The computer recorded the startle reaction for $100 \mathrm{~ms}$ after the beginning of the noise (one measure every $\mathrm{ms}$ ). These data were monitored on the computer screen.

The computer delivered a series of stimuli whose sequence and parameters had been scheduled by the computer's measurement program. After the end of the series of trials in each measurement session, the experimenter had to review each startle graph, decide whether the data it showed was valid or not. Measurements were discarded if the animal moved before delivery of the noise. He had to point with the computer mouse to the peak startle response. The amplitude had been calibrated with known weights (precision: $1 \mathrm{~g}$ ). In absence of a startle response in a quiet animal, the amplitude was quoted as 0 and included in the statistical computations.

\section{Methods specific to the first experiment}

All rats were submitted to a session lasting $30 \mathrm{~min}$. It was made of 10 series of 10 startle measurements. In each series, there were five startles alone measurements, and five startle measurements with a prepulse preceding by $100 \mathrm{~ms}$ the pulse. These conditions were randomly mixed with each other. For each rat, the mean startle level was computed as well as the percent PPI. Only the results of 33 rats were kept for the analyses because others did not show a consistent startle reaction, i.e. that occurred often enough in the pulse alone condition and that was strong enough to allow PPI measurements. The group of 33 rats was subdivided into three groups of 11 rats; low, medium and strong startling rats. The mean startle levels and the percent PPI were submitted to a between group analysis of variance, the grouping factor being the startling level groups defined above (three levels). Appropriate post hoc tests were also conducted. A statistical analysis of the baseline startle level was used to assess the validity of the threefold classification used. PPI measurements would assess how PPI depended on this classification. 


\section{Results}

The statistical analysis showed that the three levels of startle differed from one group to another $F(2,30)=27.93, P<0.0001$. A post hoc Fischer test indicated that the startle levels differed between all pairs of groups (high versus medium startle level, $P<0.0001$; high versus low startle level, $P<0.001$; low startle versus medium startle level, $P<0.05)$. The statistical analysis showed also that the percent PPI differed according to the three classes of startle levels: $F(2,30)=5.42, P<0.01$. A post hoc Fischer test indicated that PPI differed between the strong startle group and the medium one, $P<0.05$, as well as between the former and the low startle group, $P<0.01$, but not between the low startle group and the medium one, $P>0.7$. Bar graphs in the right of Fig. 2 illustrate these findings. The two bar graphs (actual data) have to be compared to the two bar graphs in the right of Fig. 1 (model). For the strongest startle, the PPI was weaker than for others.
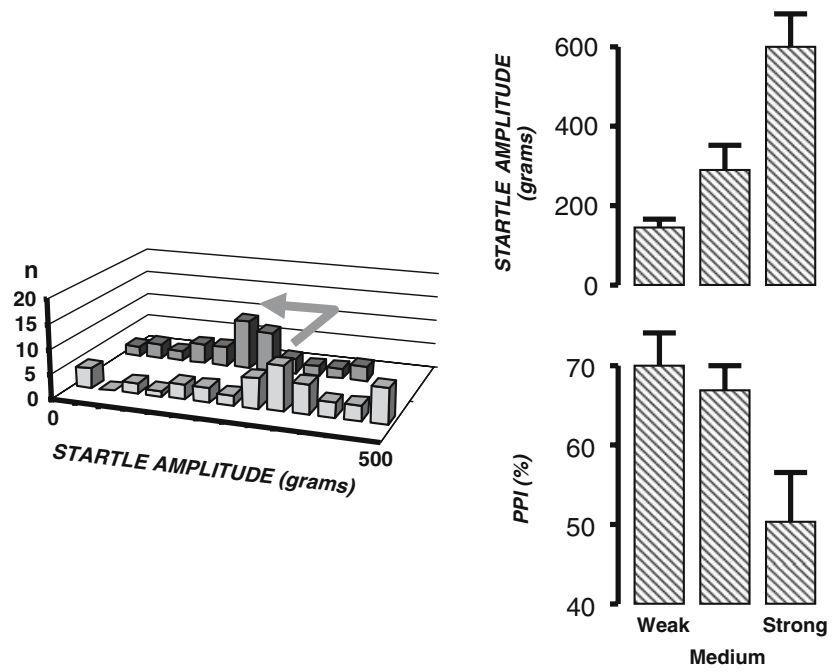

Fig. 2 Graphs representing mean + SEM startle amplitudes and percent PPI from the experimental assessment of the validity of the model. The bar graph at the left shows a typical example of the distribution of startles obtained with one rat. The baseline startle is represented as the foreground (light gray bars) and the startle with prepulses at the background (dark gray bars). The $x$-axis represents the startle level measured and the $y$-axis, the number of observations for each level. The gray arrow points to the mean startle level decrease elicited by the prepulse. At the right of the figure, bar graphs represent the resulting mean startle levels + SEM (top graph) and percent PPI + SEM (bottom graph), respectively, for weak, medium, and strong startle responses

\section{Verification of a prediction of the model}

\section{Introduction}

This series of behavioral experiment, called "other experiments" in the section labeled "methods common to all behavioral experiments", replicated at first a finding showing that an increase of PPI occurs along with the habituation to the startling noises (Ison et al. 1973; 1997; Blumenthal 1997). According to the model, we interpreted this as the manifestation of a fast habituation to the startling noise which was hidden in the startle alone condition because of a ceiling effect. This was tested in the subsequent series of behavioral experiments.

A preliminary experiment was conduced to find out the interval between prepulse and pulse that produced the strongest PPI in the strain of rats used. In order to equate the sensory experience of rats in all groups, a control group was added in which the prepulse-pulse interval was set at a very large value, known to be out of the range where PPI can occur (2000 ms). Ison et al. have shown that stabilization of startle was obtained after four to five trials (Ison et al. 1973). We limited the investigations to the initial ten trials. For the main experiment, we tested the startle after prepulse-pulse sessions, and after pulse alone sessions to avoid any interference between the startle tests with and without prepulses.

A complementary experiment was added to verify the contribution of habituation to the initial decline of the PPI startle response. It was assessed by a transient impairment of the effect of habituation as a result of a strong interfering visual stimulus. Enhancement of the effect of the prepulse over the course of the initial trials of a PPI test could result from an enhanced attention paid to it, for example as a result of a conditioned association between prepulse and pulse (Crofton et al. 1990; Flaten and Hugdahl 1990). Alternately, habituation to the pulse has been much studied using the startle reflex (Piltz and Schnitzler 1996; Sanford et al. 1992). If the observed decline in startle in the PPI condition resulted from habituation, it should share its properties with those of habituation. Thomson and Spencer have shown that submitting an animal to a strong stimulus which is different from that to which habituation has been obtained caused a transient suspension of habituation (Groves and Thompson 1970; Thompson and Spencer 1966). Thus, in the complementary experiment, we submitted rats to a brief and strong light stimulation after the 5th trial. 
Methods specific to these behavioral experiments

\section{Procedure of the preliminary experiment}

Sixty-four rats were submitted to a single test session which started as soon as the animal was placed in the test cage. It comprised 10 trials, each of which were separated from the next by a random period lasting 10-20 s. The same startling stimulus was used throughout the trials. The trials consisted of pulse alone (reference level group, $n=21$ ) or prepulse followed by the pulse with $2000 \mathrm{~ms}$ IPI (control group, $n=11$ ) and 20, 60 and $100 \mathrm{~ms}$ for test groups (respectively $n=10$, one rat being discarded, 11 and 11).

Mean startle amplitudes were submitted to a mixed design ANOVA, with the 10 measurements as a within-trial factor (10 levels) and the prepulse-pulse interval as grouping factor (five levels). The level of significance was set at $P<0.05$.

\section{Procedure of the main experiment}

Forty-eight rats were used. The experimental conditions were modified between the 5th and 6th trial to separate the circumstances of the initial habituation period from those of subsequent PPI tests. The reference startle levels were obtained by using the same procedure as in the preliminary experiment. The test group heard five pulses alone followed by five pulses preceded by prepulses, i.e. the PPI condition. Two controls groups were studied. In one of them the session started with five prepulses alone while in the other one, no stimulation was delivered during this initial period.

The prepulse-pulse interval was set at $60 \mathrm{~ms}$ according to the results of the preliminary experiments. Four groups of rats were used ( $n=12$ for each). Group $\mathrm{R}$ (Reference) was submitted to the same procedure as in the preliminary experiment. It underwent 10 trials with the prepulse-pulse sequence. Group T (Test) was submitted to five pulse-alone followed by five PPI trials. Group $\mathrm{C}_{\mathrm{pp}}$ (Control prepulse) was submitted to five prepulse-alone followed by five PPI trials. Group $\mathrm{C}_{\mathrm{ns}}$ (Control not stimulated) was placed in the cage during the same duration as the initial five trials but without any stimulus and then submitted to five PPI trials.

The mean startle amplitudes were submitted to mixed design ANOVAs. The startle amplitudes corresponding to the first five measurements was a within trial factor (five levels). The mean startle amplitudes for $\mathrm{T}$ and $\mathrm{R}$ groups were a between group factor (two levels). Furthermore, a within trial factor corresponding to the 5th and 6th measurements (two levels) was also considered, together with the experi- mental group R versus $\mathrm{T}$ factor (two levels). Similar comparisons were conducted between groups $\mathrm{C}_{\mathrm{pp}}$ and $\mathrm{C}_{\mathrm{ns}}$.

\section{Procedure of the complementary experiment}

Thirty-six male Long-Evans rats were used. They constituted three groups ( $n=12$ for each). Group $\mathrm{R}$ rats (Reference) underwent 10 trials with the "pulsealone" sequence. Group C rats (Control) underwent 10 trials with the "prepulse-pulse" sequence. Group L rats were submitted to a flashing light which was produced by repeatedly switching on and off a lamp in the test cage between the 5th and 6th trials. The lamp was switched on and off 30 times, once every $3 \mathrm{~s}$ with light on during $1.5 \mathrm{~s}$, the usual bulb replaced by a stronger $60-\mathrm{W}$ bulb. The mean startle amplitudes were submitted to a mixed design ANOVA. The 5th and 6th startle amplitude measurement was a within-trial factor (two levels) and the presence of the light flashes was a between group factor (two levels: C versus L).

Results of this series of behavioral experiments

\section{Results of the preliminary experiment}

Statistical analyses showed that the five conditions differed $F(4,59)=10.61, \quad P<0.0001$. There was a significant change in startle amplitude along the sequence of trials: $F(9,531)=5.05, P<0.0001$. This trend was not the same for every prepulse-pulse interval condition. The interaction between the prepulse-pulse interval and trial factors was significant: $F(36,531)=1.54, \quad P<0.05$. Figure 3 shows startle reactions recorded along the initial 10 measurements. They are presented separately on four graphs labeled "a, b, c and d", the same pulse-alone group being reproduced on each of them. Figure 3 a compares the two control situations, i.e. the pulse-alone condition and the condition in which prepulses occurred long before the noise (control for PPI). Startle responses remained stable from the 1 st to the 10th trial, as evidenced by the quasi-identical and horizontal regression lines in both conditions. In Fig. 3b, c, d the noise was preceded by the tone by 100,60 and $20 \mathrm{~ms}$, respectively. Mean amplitudes of the startle responses were lower than for the controls. This corresponds to the expression of PPI, which appeared especially strong for the $60 \mathrm{~ms}$ prepulse-pulse interval condition (Fig. 3c). The trend for the startle levels was a sharp decrease under the PPI conditions, i.e. an enhanced PPI effect, followed by a steadier decrease. This is visible on graphs $3 \mathrm{~b}, 3 \mathrm{c}$ and $3 \mathrm{~d}$. 

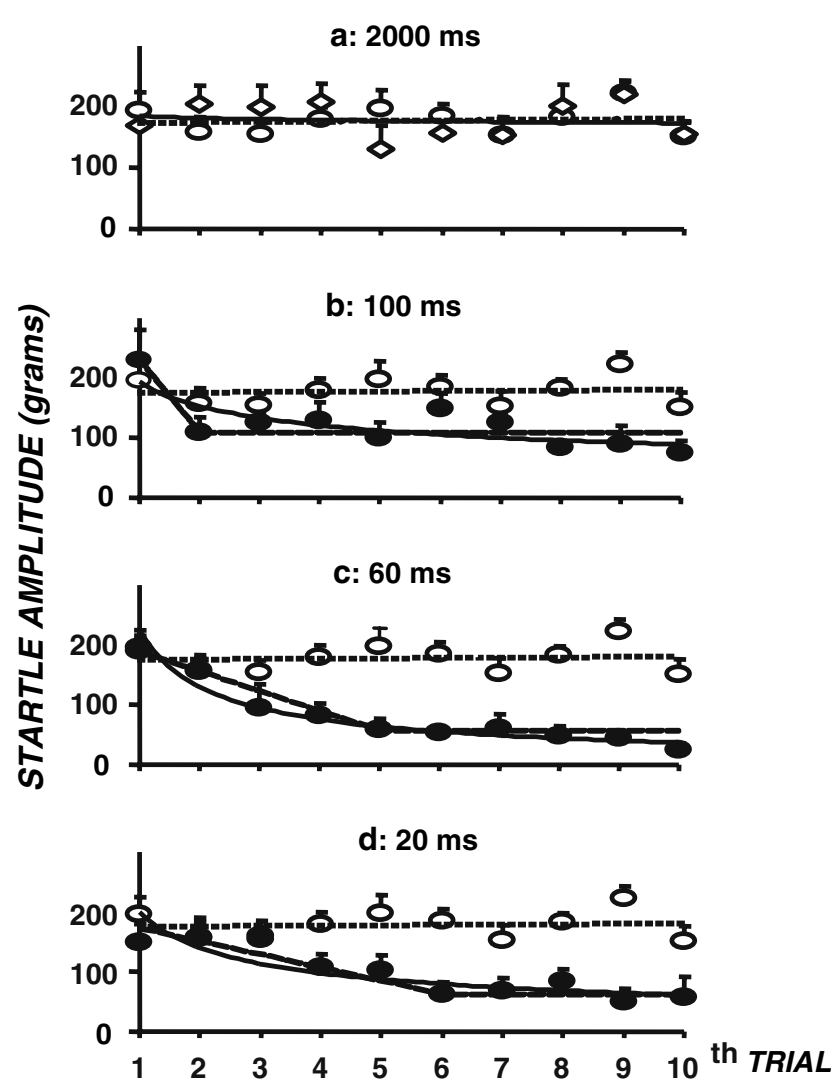

Fig. 3 Graphs representing mean + SEM startle amplitudes as a function of the sequence of trials for a number of IPIs. Each graph comprises the reference startle group, whose data were plotted as clear circles. Graph a shows as diamonds the data from the control group where the IPI was $2000 \mathrm{~ms}$, i.e. too long to elicit any PPI effect. Graphs $\mathbf{b}$, $\mathbf{c}$, and $\mathbf{d}$ show as black circles the experimental data for startle reduced by the PPI effect with an IPI of 100, 60, and $20 \mathrm{~ms}$, respectively (values rounded up for clarity, being actually 110,62 , and $22 \mathrm{~ms}$ for technical reasons as they are binary subdivisions of an electronic clock). Each graph comprises a dotted line corresponding to the regression line of the reference group and an unbroken line corresponding to a power function regression through each of the experimental data. A dashed line was also added to indicate an alternative authors' interpretation of the data

\section{Results of the main experiment}

Statistical analyses revealed that the first five trials of groups $\mathrm{T}$ and $\mathrm{R}$ differed: Group factor: $F(1,22)=22.25, P<0.001$. The sudden change in test conditions between the 5 th and the 6 th trial elicited a significant change in responsiveness: $F(1,11)=51.32$, $P<0.0001$. The last five trials of groups $C_{\mathrm{pp}}$ and $\mathrm{C}_{\mathrm{ns}}$ were far from showing any difference: Group factor: $F(1,21)=0.85$. Figure $4 \mathrm{a}$ shows the progressive increase of PPI in group R similar to the preliminary experiment, except that it looks shorter (three trials rather than four). The first five trials for group $\mathrm{T}$ shows the same aspects as the controls of the preliminary experiment, i.e. minimum decrease in startle as trials were repeated. After the 5th trial, there appeared a sudden change in responsiveness, highlighted by the gray arrow on the graph. The graph corresponding to group $\mathrm{T}$ suddenly becomes like that of group $\mathrm{R}$. Figure $4 \mathrm{~b}$ shows that, after five trials, where no stimulus was applied or where the tone serving as a prepulse was applied alone, both graphs look like the beginning of the graph corresponding to group R, above.

\section{Results of the complementary experiment}

The sudden change in test conditions between the 5 th and the 6th trial elicited a significant change in startle responsiveness: $F(1,21)=4.60, P<0.05$. The bar graph in Fig. 5 shows the amplitude levels before the light stimulus (L5) and just after (L6), compared to the reference levels (R5 and R6) and control levels (C5 and C6). There was an increase between the 5th and the 6th trial for the test group, whereas bars C5 and C6 show that a decrease or no change should have occurred. The effect was transient as no more visible on

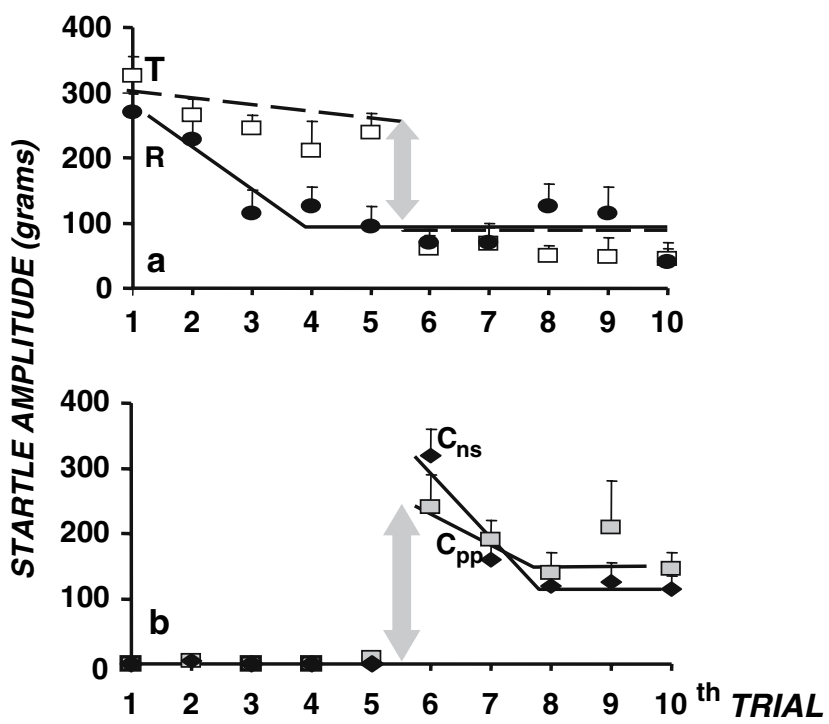

Fig. 4 Graphs representing mean + SEM startle amplitudes as a function of the sequence of trials when the test conditions were suddenly changed between the 5th and 6th trials (except for one group). In all graphs, trials 6-10 corresponded to the same PPI condition. In graph a, the clear squares and dashed broken line stand for the group, in which pulse alone was applied from trial 1 to $5(\mathrm{~T})$. The black dots represent the control group in which the PPI conditions were applied along all the trials (R). In graph $\mathbf{b}$, the black diamonds stand for the group, in which no stimulation was applied in trials 1-5 $\left(\mathrm{C}_{\mathrm{ns}}\right)$ and the darkened squares stand for the group, in which a prepulse alone was applied in trials 1-5 $\left(\mathrm{C}_{\mathrm{pp}}\right)$. The gray arrows highlight the transition between the 5 th and 6 th trial 


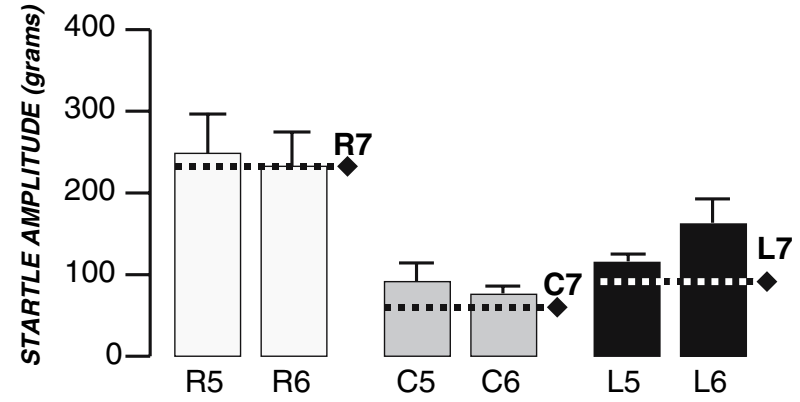

Fig. 5 Bar graphs representing mean + SEM startle amplitudes corresponding to the measurements conducted in the 5th and 6th trials in the experiment on dishabituation. The clear bars represent the reference group submitted to the pulse alone in their 5th and the 6th trials (R5 and R6). The darkened bars represent the control group submitted to the prepulse-pulse sequence during the same trials (C5 and C6). The black bars represent the result of a group submitted to the same stimuli as group $\mathrm{C}$ but with light flashes inserted between the two trials. For completeness, the level corresponding to the subsequent trial (the 7th, R7, C7 and L7) is indicated by a dotted line

the next measurement, as indicated by the dotted line (holding respectively for $\mathrm{R} 7, \mathrm{C} 7$, and L7).

Discussion of this series of behavioral experiments

\section{Discussion of the preliminary experiment}

On its first assessment, the startle level was not sensitive to the presence of a prepulse. This is in contradiction to previous observations in our laboratory where a PPI occurred as soon as the first trial, albeit under different conditions and in another species of rat (Canal et al. 2001). PPI obtained as soon as on its first trial could be attributed to rats having had previous experiences of strong sounds. Even if rats were maintained in a carefully controlled environment in their housing room, they would certainly have heard stressful noises during their journey from the rat farm to the laboratory. Under the $60 \mathrm{~ms}$ prepulse-pulse interval condition, PPI became stable after less than six trials. Such a result confirms and extends previous observations (Ison et al. 1973) as it was the condition producing the strongest PPI. This justified the use of this parameter for the main experiment.

\section{Discussion of the main experiment}

There were some minor differences between the reference group in this experiment and the results of the preliminary experiment. The startle amplitudes were somewhat stronger ( $275 \mathrm{~g}$ versus $200 \mathrm{~g}$ at the first trial). The difference can be attributed to the slight methodological change between both experiments, as mentioned in the methods chapter. But even when similar methods were used, such differences occurred along other experiments in our laboratory from one set of rats to another. Another difference was that only three trials were required to reach an asymptotic startle in PPI conditions in this experiment compared to four trials in the previous one. But this depends on the subjective interpretation of the elbow of a curve drawn through data. A third difference consisted in the occurrence of a slight but visible decline in the graph of the controls in the main experiment. This aspect of the results, even if the differences among strong startle levels remain below the statistically detectable threshold in the absence of the reduction elicited by PPI are well in line with our hypothesis of a hidden habituation in the absence of prepulse. Irrespective of these minor differences between both experiments, maximum PPI was obtained when the rat had been submitted to a series of baseline startle tests. In other words, the startle eliciting noise was the only factor that produced the decline in the startle responses visible only in PPI conditions.

\section{Discussion of the complementary experiment}

There was a decline in startle in the initial course of PPI measurements in our experimental conditions attributed to habituation to the startle eliciting stimulus. The search for the possibility to erase transiently the habituation as proposed by Thompson and Spencer has been successful (Thompson and Spencer 1966). It was moderate and transient. This is not surprising as it depends on the strength of the interfering stimulus. That one used in this experiment was weak compared to the electrical shocks used in other laboratories. Nevertheless, the occurrence of a significant effect provided an argument in favor of the involvement of habituation in the decline of the initial startle responses in the presence of a prepulse (PPI condition).

\section{Conclusion of this series of behavioral experiments}

Through the preliminary experiment, temporal parameters were established that made it possible to obtain reproducible observations of a rapid increase in PPI (decline of startle in PPI conditions together with a stationary baseline startle). It required between one and four trials depending on the parameters used. The main experiment showed that the loud startling noise was the only factor of this alteration of PPI. The time course of the startle reactions when there was a prepulse resembled an habituation to noise. But there 
was no directly observable effect on the baseline startle in the same parametric conditions. The involvement of a habituation process was confirmed using a "dishabituation" experiment. This showed that a property of the baseline startle could be revealed in PPI conditions, even if it has no relationship with PPI itself.

\section{General discussion}

Leumann et al. (2001) modeled the acoustic startle reflex and PPI using neural network simulation methods. But, neither the relationship between PPI and the startle levels, nor the variability of the baseline startles, nor habituation have been considered in their published works. Schmajuk and Larrauti (2005) and Schmajuk et al. (2006) proposed a model that put the emphasis on the role of three convergent influences. There are four differences between their model and ours. At first, a dynamic aspect was considered in their work which was left out of the present study. The second difference resides in the transfer functions selected. For the upper limits of such functions, there may be only minor consequences of this difference. But, for the lower values, the output (E2 in equation 10, Schmajuk and Larrauri 2005) tends to a finite value in Schmajuk et al.'s model, whereas we considered an horizontal asymptote. This may be of importance only for low PPI values. Considering individual startles that vary from one moment to another was something original in the present study. Even for a strongly responsive animal, very low startle levels occur from time to time and contribute to the mean startle level computations. As a consequence, asymptotical properties of transfer functions contribute to the means startle level. The fourth and major difference resides in the interaction between excitations and inhibitions. It was an additive one in Schmajuk et al.'s model. But in our model, the gain of the input-output relationship was reduced in the presence of a prepulse. PPI decreased when startle increased in our behavioral experiments. It had been predicted both by our model and that one of Schmajuk et al. But there is a slight difference between the predictions of Schmajuk et al.'s model and our experimental data as well as those of Yee et al. (2005). There was a larger PPI for the medium range of baseline startle level. This fits better with the prediction of our model. Playing with the equations of the model of Schmajuk and Larrauti (2005) and Schmajuk et al. (2006), or with the excitation/inhibition rules, should be able to account for this minor difference of prediction of both models (Fig. 6).
A number of experiments used strong noises to trigger the startle reaction. They were assumed to model what happens in schizophrenic patients. The results of the present work point: (i) the possibility of misleading distortions when using strong startling noises and (ii) indicate that PPI and baseline startle measurements are not independent. While we showed that the initial decrease of PPI resulted from the rat's prior experience of the strong startling noise, we do not know whether this effect is specific to the strong noise itself or is related to its stressful, aversive, effect. This could be verified by conducting the same experiment with an air-puff eliciting the startle response (Taylor et al. 1991) during the first five trials and noise afterwards. If stress plays a role irrespective of the sensory modality then a cross stimulus modality PPI modification would occur. This is a significant issue for further studies for at least two reasons: the relationship between the anxiogenic effect of a stressful event and PPI needs to be better documented-in lower animal species as well as in humans-and it is worth exploring the contribution of stress to the management of the attention paid to a stimulus used as prepulse. Habituation of the rat to strong sounds was our alternate explanation. It was reinforced by the observations. But, there are some contradictory observations to consider in the literature (Gewirtz and Dawis 1995; Lipp and Krinitzky 1998). In their studies, PPI was found to decrease rather than increase. A strong habituation in the pulse-alone condition happened in such experiments. It would help to understand the contradiction as the relative efficiency of the prepulse

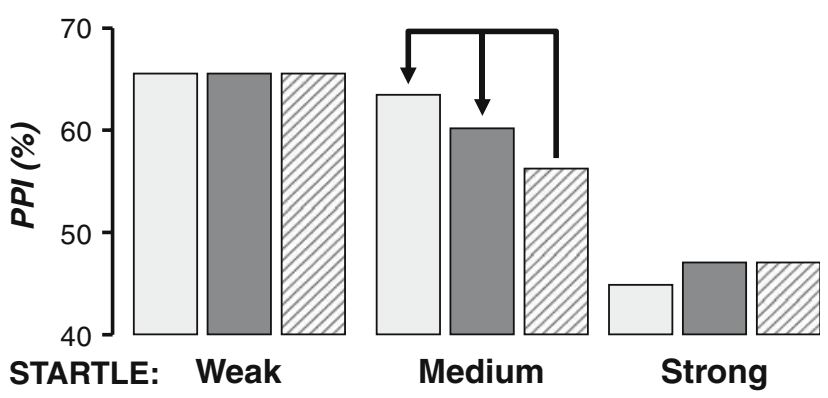

Fig. 6 Bar graphs representing the results of PPI computations done with the data plotted on figure 11 of Schmajuk and Larrauri (2005). The bar graphs represent the percent PPI for weak, medium, and strong startling influences from our experiment (light gray bars: reproduction of the bottom right bar graph of Fig. 1), an experiment of Yee et al. (2005) (dark gray bars: data for which the representation has been expressed in the same standard way as ours), and the results of Schmajuk and Larrauri's model (hached bars: enhanced by $7.5 \%$ ). The fit is good for the weak and strong inputs but the model data depart from experimental ones for medium levels of stimulation (as pointed by the arrows) 
would have been strengthened. It means that, according to the parametric setting of the experiments, an increase or a decrease of PPI may happen, both expressing a linkage between PPI and baseline startle, which is the main topic of the present paper. This argues for the necessity of systematic parametric approaches of startle and PPI. The link between habituation and PPI change mentioned above reminds that habituation was also altered in schizophrenia (Braff et al. 2001; Swerdlow et al. 1995) even if this remains controversial (Ludewig et al. 2002; Mackeprang et al. 2002). Furthermore, habituation and PPI were found to share a number of pharmacological properties (Geyer et al. 1990).

Human and lower animal research on the properties of the startle reaction, for example, (i) its evolution and variability along iterative measurements, (ii) its sensitivity to sensory events that happen a few milliseconds before the startle eliciting stimulus, i.e. PPI, (iii) the nature and parametric diversity of stimuli use, and (iv) its sensitivity to the emotional circumstances will still require a considerable amount of work to be able to solve the contradictions of the literature. Models are helpful not only to resolve apparent discrepancies in the literature but also point on the parameters that are the more relevant to include in the studies and to understand pathophysiological observations.

Acknowledgments Mrs. Nathalie Monique Canal was granted by the French Ministry of Education. We thank Pr. Terence O'Brien for his suggestions concerning the last version of the manuscript, especially for ameliorating its English version. The stylistic aspect of its initial versions had been verified by Mr. Andrew Wright, translator of the European Parliament.

\section{References}

Blumenthal TD (1997) Prepulse inhibition decreases as startle reactivity habituates. Psychophysiology 34: 446-450

Braff DL, Geyer MA, Swerdlow NR (2001) Human studies of prepulse inhibition of startle: normal subjects, patient groups, and pharmacological studies. Psychopharmacology 156: $234-258$

Canal NM, Gourevitch R, Sandner G (2001) Non monotonic dependency of PPI on temporal parameters: differential alteration by ketamine and MK-801 as opposed to apomorphine and DOI. Psychopharmacology 156: 169-176

Crofton KM, Dean KF, Sheets LP, Peele DB (1990) Evidence for an involvement of associative conditioning in reflex modulation of the acoustic startle response with gaps in background noise. Psychobiology 18: 467-474

Davis M (1970) Effects of interstimulus interval length and variability on startle response habituation in the rat. J Comp Physiol Psychol 72: 177-192

Davis M (1974) Sensitization of the rat startle response by noise. J Comp Physiol Psychol 87: 571-581
Davis M (1989) Sensitization of the acoustic startle reflex by footshock. Behav Neurosci 103: 495-503

de Lima TC, Davis M (1995) Involvement of cyclic AMP at the level of the nucleus reticularis pontis caudalis in the acoustic startle response. Brain Res 700: 59-69

Flaten MA, Hugdahl K (1990) Effect of habituation and classical conditioning on reflex modification. Int J Psychophysiol 9: 129-137

Geyer MA, Swerdlow NR, Mansbach RS, Braff D (1990) Startle response models of sensorimotor gating and habituation deficits in schizophrenia. Brain Res Bull 25: 485-498

Geyer MA, Thomson KK, Braff DL, Swerdlow NR (2001) Pharmacological studies of prepulse inhibition models of sensorimotor gating deficits in schizophrenia: a decade in review. Psychopharmacology 156: 117-154

Gewirtz JC, Dawis M (1995) Habituation of prepulse inhibition of the startle reflex using an auditory prepulse close to background noise. Behav Neurosci 109: 388-395

Graham FK (1975) The more or less startling effects of weak prestimulation. Psychophysiology 12: 238-48

Groves PM, Thompson, RF (1970) Habituation: a dual-process theory. Psychol Rev 77: 419-450

Groves PM, Boyle RD, Welker RL, Miller SW (1974) On the mechanism of prepulse inhibition. Physiol Behav 12: 367-75

Ison JR, Hammond GR, Krauter EE (1973) Effects of experience on stimulus-produced reflex inhibition in the rat. J Comp Physiol Psychol 83: 324-336

Ison JR, Bowen GP, Pak J, Gutierrez E (1997) Changes in the strength of prepulse inhibition with variation in the startle baseline associated with individual differences and with old age in rats and mice. Psychobiology 25: 266-274

Johansson C, Jackson DM, Zhang J, Svensson L (1995) Prepulse inhibition of acoustic startle, a measure of sensorimotor gating: effects of antipsychotics and other agents in rats. Pharmacol Biochem Behav 52: 649-654

Koch M (1999) The neurobiology of startle. Prog Neurobiol 59: 107-128

Koch M, Kungel M, Herbert H (1993) Cholinergic neurons in the pedunculopontine tegmental nucleus are involved in the mediation of prepulse inhibition of the acoustic startle response in the rat. Exp Brain Res 97: 71-82

Krase W, Koch M, Schnitzler HU (1993) Glutamate antagonists in the reticular formation reduce the acoustic startle response. Neuroreport 4: 13-16

Le Pen G, Moreau JL (2002) Disruption of prepulse inhibition of startle reflex in a neurodevelopmental model of schizophrenia: reversal by clozapine, olanzapine and risperidone but not by haloperidol. Neuropsychopharmacology 27: 1-11

Leumann L Sterchi D, Vollenweider F, Ludewig K, Fruh H (2001) A neural network approach to the acoustic startle reflex and prepulse inhibition. Brain Res Bull 56: 101-10

Lipp OV, Krinitzky SP (1998) The effect of repeated prepulse and reflex stimulus presentations on startle prepulse inhibition. Biol Psychol 47: 65-76

Lipska BK, Swerdlow NR, Geyer MA, Jaskiw GE, Braff DL, Weinberger DR (1995) Neonatal excitotoxic hippocampal damage in rats causes post-pubertal changes in prepulse inhibition of startle and its disruption by apomorphine. Psychopharmacology (Berl) 122: 35-43

Ludewig K, Geyer MA, Etzenberger M, Vollenweider FX (2002) Stability of the acoustic startle reflex, prepulse inhibition, and habituation in schizophrenia. Schizophr Res 55: 129-137

Mackeprang T, Kristiansen KT, Glenthoj BY (2002) Effects of antipsychotics on prepulse inhibition of the startle response in drug-naive schizophrenic patients. Biol Psychiatry 52: $863-873$ 
Nodal FR, Lopez DE (2003) Direct input from cochlear root neurons to pontine reticulospinal neurons in albino rat. J Comp Neurol 460: 80-93

Piltz PKD, Schnitzler HU (1996) Habituation and sensitization of the acoustic startle response in rats: amplitude, threshold, and latency measures. Neurobiol Learn Mem 66: 67-79

Sanford LD, Ball WA, Morrison AR, Ross RJ, Mann G (1992) Peripheral and central components of alerting: habituation of acoustic startle, orienting response, and elicited waveforms. Behav Neurosci 106: 112-120

Schmajuk NA, Larrauri JA (2005) Neural network model of prepulse inhibition. Behav Neurosci 119: 1546-62

Schmajuk NA, Larrauri JA, Hagenbuch N, Levin ED, Feldon J, Yee BK (2006) Startle and prepulse inhibition as a function of background noise: A computational and experimental analysis. Behav Brain Res 170: 182-96

Swerdlow NR, Filion D, Geyer MA, Braff DL (1995) "Normal" personality correlates of sensorimotor, cognitive, and visuospatial gating. Biol Psychiatry 37: 286-299

Swerdlow NR, Geyer MA (1998) Using an animal model of deficient sensorimotor gating to study the pathophysiology and new treatments of schizophrenia. Schizophr Bull 24: 285-301

Taylor BK, Carsto R, Printz MP (1991) Dissociation of tactile and acoustic components in air puff startle. Physiol Behav 49: 527-532

Thompson RF, Spencer WA (1966) Habituation: a model phenomenon for the study of neuronal substrates of behavior. Psychol Rev 73: 16-43

van Koppen CJ, Kaiser B (2003) Regulation of muscarinic acetylcholine receptor signaling. Pharmacol Ther 98: 197-220

Yee BK, Chang T, Pietropaolo S, Feldon J (2005) The expression of prepulse inhibition of the acoustic startle reflex as a function of three pulse stimulus intensities, three prepulse stimulus intensities, and three levels of startle responsiveness in C57BL6/J mice. Behav Brain Res 163: 265-276

Yeomans JS, Frankland PW (1995) The acoustic startle reflex: neurons and connections. Brain Res Brain Res Rev 2: 301-314 